\title{
AVALIAÇÃO DA TRANSMISSÃO DE DADOS DE TEMPERATURA NO SISTEMA 1-WIRE ${ }^{\mathrm{TM} 1}$
}

\section{ANTONIO J. STEIDLE NETO ${ }^{2}$, FERNANDO C. BAÊTA ${ }^{3}$, JOSÉ H. MARTINS ${ }^{4}$, SÉRGIO ZOLNIER ${ }^{4}$, PAULO M. B. MONTEIRO ${ }^{5}$}

\begin{abstract}
RESUMO: A necessidade de sistemas de monitoramento automático versáteis e de baixo custo que possam auxiliar o produtor agrícola na otimização dos processos produtivos, é evidente. Este trabalho foi realizado com o objetivo de pesquisar as limitações e as potencialidades de aplicação do sistema 1wire $^{\mathrm{TM}}$ na transmissão de dados de temperatura em instalações agrícolas. O sistema 1 -wire ${ }^{\mathrm{TM}}$ é uma rede de transmissão de dados que possibilita a comunicação digital entre um computador e dispositivos da série 1-wire ${ }^{\mathrm{TM}}$, tais como os sensores de temperatura DS1820. A transmissão de dados de temperatura nesse sistema foi avaliada em função do tipo dos condutores e do número de sensores DS1820. Com base nos resultados, concluiu-se que o aumento do número de sensores de temperatura DS1820 no sistema 1-wire ${ }^{\mathrm{TM}}$ incrementa a carga capacitiva de maneira distinta para cada um dos tipos de condutores estudados, podendo causar interrupções na transmissão de dados.
\end{abstract}

PALAVRAS-CHAVE: sistemas de aquisição de dados, sensores de temperatura, instalações agrícolas.

\section{EVALUATION OF THE TEMPERATURE DATA TRANSMISSION IN THE 1-WIRE ${ }^{\text {TM }}$ SYSTEM}

\begin{abstract}
The need of versatile and low cost automatic monitoring systems for the optimization of the agricultural productive processes is evident. This work was carried out to evaluate the limitations and potentialities of the 1-wire ${ }^{\mathrm{TM}}$ system for temperature data transmission in agricultural buildings. The 1-wire ${ }^{\mathrm{TM}}$ system is a data transmission network, which makes possible the digital communication between a computer and devices of the 1-wire ${ }^{\mathrm{TM}}$ series, such as the temperature DS1820 sensors. The temperature data transmission in this system was evaluated as a function of types of conductors and the number of DS1820 sensors. Based on the results, it was concluded that, by increasing the number of the DS1820 temperature sensors in the 1-wire ${ }^{\mathrm{TM}}$ system, the capacitive load increases in a different way for each conductor and can cause interruptions during temperature data transmission.
\end{abstract}

KEYWORDS: data acquisition systems, temperature sensors, agricultural buildings.

\section{INTRODUÇÃO}

O ambiente interno às instalações agrícolas no qual as espécies de interesse são submetidas, sejam elas animais, sejam vegetais, constitui um dos principais fatores responsáveis pelo sucesso ou fracasso do empreendimento agrícola. Segundo CURTIS (1983), esse ambiente pode ser definido como a integração de variáveis químicas, biológicas e climáticas circundantes. As variáveis climáticas, como as demais variáveis mencionadas, atuam e interagem influenciando no desempenho das espécies. A temperatura do ar destaca-se por apresentar papel relevante, pois, associada a outros fatores, pode

\footnotetext{
${ }^{1}$ Extraído da Dissertação de Mestrado do primeiro autor

${ }^{2}$ Eng ${ }^{\mathrm{o}}$ Agrícola, Doutorando em Engenharia Agrícola, Departamento de Engenharia Agrícola, Universidade Federal de Viçosa, Viçosa MG, Fone: (0xx31) 3899.2734, staidle@ hotmail.com

${ }^{3}$ Eng $^{0}$ Agrônomo, Vice-Reitor, Prof. Doutor, Departamento de Engenharia Agrícola, UFV, Viçosa - MG.

${ }^{4}$ Eng ${ }^{0}$ Agrícola, Prof. Doutor, Departamento de Engenharia Agrícola, UFV, Viçosa - MG.

${ }^{5}$ Eng ${ }^{0}$ Eletrônico e de Telecomunicações, Prof. Doutor, Departamento de Engenharia de Controle e Automação, UFOP, Ouro Preto-MG. Recebido pelo Conselho Editorial em: 22-12-2003

Aprovado pelo Conselho Editorial em: 21-2-2005
} 
comprometer ou favorecer o crescimento e o desenvolvimento em função das exigências de cada espécie.

A principal aplicação do monitoramento automático em instalações agrícolas consiste na possibilidade de controle do ambiente interno por meio de atuadores, como ventiladores, exaustores, nebulizadores, painéis de resfriamento evaporativo, dentre outros, que são acionados em resposta a determinadas condições consideradas desfavoráveis, promovendo intervenções específicas (STEIDLE NETO, 2003). Dentre os principais entraves ao monitoramento automático de instalações agrícolas destinadas à produção de animais ou vegetais, em escala industrial, destacam-se os altos custos dos sistemas de aquisição de dados.

O sistema 1-wire ${ }^{\mathrm{TM}}$ desenvolvido pela empresa Dallas Semiconductor tem se mostrado promissor em aplicações agrícolas. Esse sistema trata-se de uma rede de transmissão de dados que possibilita a comunicação digital entre um computador e dispositivos da série 1-wire ${ }^{\mathrm{TM}}$, tais como sensores e adaptadores. De acordo com AWTREY (2001), o sistema 1-wire ${ }^{\mathrm{TM}}$ é constituído, basicamente, por três elementos: um computador com programa de controle e gerenciamento das atividades, seguindo um protocolo específico de comunicação, condutores (meio físico de comunicação) e dispositivos remotos da série 1-wire ${ }^{\mathrm{TM}}$.

Normalmente, a transmissão de dados ocorre por meio de um condutor e, além desse, existe um condutor específico para fornecer energia aos sensores e outro para o aterramento da rede. Por definição, o sistema 1-wire ${ }^{\mathrm{TM}}$ possui apenas um condutor no qual são conectados todos os dispositivos da série 1-wire ${ }^{\mathrm{TM}}$, já que, por convenção, o condutor de referência ou aterramento não é considerado. $\mathrm{O}$ fornecimento de energia para o sistema 1-wire ${ }^{\mathrm{TM}}$ pode ser de duas maneiras: alimentação parasita (derivada do condutor de transmissão de dados), sendo necessário somente dois condutores, ou alimentação externa em que são necessários três condutores, além de uma fonte de alimentação regulada de 5 Vcc.

Uma condição prévia para o funcionamento de qualquer rede de transmissão de dados que empregue diversos dispositivos semelhantes é a existência de endereços ou códigos de identificação. Todo dispositivo 1-wire ${ }^{\mathrm{TM}}$ possui um código de identificação único de 64 bits seqüenciais, o que exclui a possibilidade de conflitos durante a transmissão de dados em redes com diversos dispositivos.

$\mathrm{O}$ protocolo de comunicação do sistema 1 -wire ${ }^{\mathrm{TM}}$ utiliza níveis lógicos convencionais CMOS/TTL, no qual o nível lógico 0 (zero) é representado por uma tensão máxima de 0,8 Vcc e o nível lógico 1 (um) por uma tensão mínima de 2,2 Vcc (DALLAS SEMICONDUCTOR, 2002 b).

MONTEIRO (2002) desenvolveu um sistema de controle inteligente para aeração de grãos armazenados, com base na tecnologia 1-wire ${ }^{\mathrm{TM}}$ desenvolvida pela Dallas Semiconductor, utilizando sensores de temperatura DS1820. Os resultados obtidos nos ensaios realizados em um protótipo de silo confirmaram a simplicidade e o excelente desempenho dessa tecnologia nesse tipo de aplicação. Contudo, não foram abordadas pelo autor questões referentes ao comportamento da transmissão de dados de temperatura no sistema 1-wire ${ }^{\mathrm{TM}}$.

Visando à aplicação dessa tecnologia em instalações agrícolas, este trabalho foi realizado com o objetivo de avaliar o efeito do acréscimo de sensores de temperatura DS1820 à linha de transmissão de dados, bem como o tipo de condutor utilizado para a implementação do sistema 1-wire ${ }^{\mathrm{TM}}$.

\section{MATERIAL E MÉTODOS}

A avaliação da transmissão de dados de temperatura por meio do sistema 1-wire ${ }^{\mathrm{TM}}$ foi realizada no Laboratório de Construções Rurais e Ambiência, pertencente ao Departamento de Engenharia Agrícola da Universidade Federal de Viçosa. 
Neste trabalho, foram utilizados cinco tipos de condutores com comprimento individual de $200 \mathrm{~m}$, relacionados a seguir:

1) Cabo telefônico trançado FI $2 \times 0,24 \mathrm{~mm}^{2}$;

2) Cabo telefônico paralelo especial FI $2 \times 0,28 \mathrm{~mm}^{2}$;

3) Cabo telefônico CCI 2 x 0,20 $\mathrm{mm}^{2}$;

4) Cabo coaxial blindado (microfone mono) $0,20 \mathrm{~mm}^{2}, \mathrm{e}$

5) Cordão trançado $2 \times 1 \mathrm{~mm}^{2}$.

Além dos condutores, foram empregados 20 sensores de temperatura DS1820, previamente calibrados, um adaptador universal DS9097U-009, uma fonte de alimentação externa regulada com saída de 5 Vcc, um osciloscópio Hewlett Packard, modelo 54610B de $500 \mathrm{MHz}$, e um computador IBM 586.

Na seleção dos condutores, consideraram-se dois critérios em ordem de importância: econômico (condutores de baixo custo) e comercial (condutores comumente comercializados para fins domiciliares).

Qualquer que seja o tipo dos condutores usados na transmissão de dados, as suas características físicas, como a área da seção transversal, o comprimento e o espaçamento entre condutores, além do material isolante, conferem a esses características elétricas, como resistência, indutância e capacitância. Dentre essas características elétricas, a capacitância dos condutores é a que causa os maiores problemas na transmissão de dados entre o computador e os sensores de temperatura no sistema 1-wire $^{\mathrm{TM}}$. O mesmo pode ser afirmado em relação aos sensores de temperatura DS1820 da série 1-wire ${ }^{\mathrm{TM}}$, cuja capacitância individual é de aproximadamente 30 pF (DALLAS SEMICONDUCTOR, 2001). Assim sendo, a capacitância impõe rígidas restrições ao tipo dos condutores e ao número de sensores de temperatura DS1820.

De acordo com a DALLAS SEMICONDUCTOR (2002 a), a capacitância no sistema 1-wire ${ }^{\mathrm{TM}}$ aumenta com o acréscimo do número de sensores de temperatura DS1820 e com a capacitância dos condutores. $\mathrm{O}$ incremento da capacitância faz com que o tempo para que a linha de transmissão de dados seja levada do nível lógico 0 (zero) para o nível lógico 1 (um) aumente. Se o produto entre o valor do resistor de polarização (1.500 $\Omega$ ) interno ao adaptador universal DS9097U-009 e a capacitância total (capacitância dos condutores + capacitância dos sensores DS1820) resultar em uma constante de tempo (RC) que exceda o intervalo de tempo definido no protocolo de comunicação 1wire $^{\mathrm{TM}}$ para um determinado bit, a comunicação é interrompida. Segundo AWTREY (2002) ${ }^{*}$, a capacitância total não deve exceder a um valor máximo permitido $(\approx 10.000 \mathrm{pF})$ para que a transmissão de dados de temperatura no sistema 1-wire ${ }^{\mathrm{TM}}$ não seja interrompida.

Como os fabricantes de condutores não são obrigados, pela legislação pertinente, a realizar ensaios de laboratório a fim de determinar os valores de capacitância dos condutores por eles produzidos, foi necessário recorrer a um laboratório especializado. Assim sendo, os valores de capacitância dos condutores estudados foram determinados no Laboratório de Análise de Redes, pertencente ao Instituto de Engenharia Elétrica da Universidade Federal de Itajubá, por meio de equipamento denominado Ponte de Schering. Por se tratar de um equipamento de alta precisão, a Ponte de Schering permite erro máximo de $\pm 0,04 \%$ para medidas de capacitância.

Primeiramente, foram realizadas avaliações teóricas da transmissão de dados de temperatura, utilizando-se de modelo teórico [eq.(1)] que descreve a transição do nível lógico 0 (zero) para o nível lógico 1 (um) no sistema 1-wire ${ }^{\mathrm{TM}}$. Esse modelo considera que a tensão no condutor de transmissão de

AWTREY, D. Total capacitive load on a 1-wire ${ }^{\mathrm{TM}}$ net. Mensagem pessoal via e-mail. 17 set. 2002. 
dados se comporta de acordo com o carregamento do capacitor, cuja corrente é limitada pelo resistor de polarização.

$$
\mathrm{V}_{\mathrm{L}}(\mathrm{t})=\mathrm{V}_{\mathrm{S}}-\mathrm{V}_{\mathrm{S}} \cdot \mathrm{e}^{-\mathrm{t} / \mathrm{RC}}
$$

em que,

$\mathrm{V}_{\mathrm{L}}$ - tensão na linha de transmissão de dados, Vcc;

$\mathrm{V}_{\mathrm{S}}$ - tensão de alimentação aplicada pela fonte externa, $5 \mathrm{Vcc}$;

$\mathrm{t}$ - tempo, s;

$\mathrm{R}$ - valor do resistor de polarização, $1.500 \Omega$, e

C - capacitância total (condutores + sensores), $\mathrm{pF}$.

Com base nos gráficos obtidos a partir do modelo teórico, determinaram-se os intervalos de tempo de transição do nível lógico 0 (zero) para o nível lógico 1 (um) no sistema 1-wire ${ }^{\mathrm{TM}}$.

Para a condução das avaliações experimentais, determinou-se inicialmente o comprimento máximo de transmissão de dados para cada um dos cinco tipos de condutores, utilizando-se de um único sensor de temperatura DS1820. O comprimento máximo representa o comprimento limite dos condutores acima do qual a comunicação entre o sensor de temperatura DS1820 e o computador é interrompida ou resulta em valores de temperatura não condizentes com a temperatura real do meio onde o sensor se encontra. Em seguida, realizaram-se avaliações da transmissão de dados para cada tipo de condutor, com 1; 10 e 20 sensores de temperatura DS1820 conectados ao sistema. As imagens das formas de onda foram capturadas pelo osciloscópio, por meio de duas pontas de prova, nos instantes em que a linha de transmissão de dados era levada do nível lógico 0 (zero) para o nível lógico 1 (um). Esse procedimento teve por finalidade determinar os intervalos de tempo de transição entre os níveis lógicos zero e um. Os conjuntos de pontos gerados, a partir das imagens das formas de onda congeladas no osciloscópio, tornaram possível a reprodução dessas imagens em planilhas eletrônicas, permitindo plotar em um mesmo gráfico, para cada tipo de condutor, as três curvas de transição correspondentes a 1; 10 e 20 sensores de temperatura. Nas avaliações experimentais, foram realizadas as mesmas combinações das avaliações teóricas, permitindo, dessa maneira, comparar os tempos de transição entre níveis lógicos obtidos em ambas.

Em todas as avaliações teóricas e experimentais, considerou-se que o nível lógico um era alcançado quando a tensão no condutor de transmissão de dados atingia o patamar de 2,2 Vcc.

\section{RESULTADOS E DISCUSSÃO}

O gráfico da Figura 1 representa a condição ideal de transição entre níveis lógicos na qual a capacitância total do sistema 1 -wire ${ }^{\mathrm{TM}}$ é muito baixa, possibilitando que a transição ocorra de maneira instantânea.

O tempo de transição do nível lógico 0 (zero) para o nível lógico 1 (um) corresponde ao tempo a partir do qual a linha de transmissão de dados apresenta aumento na tensão (acima de 0 Vcc) até a tensão limite de 2,2 Vcc (linha horizontal).

Neste trabalho, serão apresentados os gráficos resultantes das avaliações teóricas e experimentais para o tipo de condutor que apresentou o melhor desempenho (cabo telefônico CCI), Figuras 2 e 3, respectivamente, e para o tipo de condutor de pior desempenho (cabo coaxial blindado), Figuras 4 e 5 , respectivamente. 


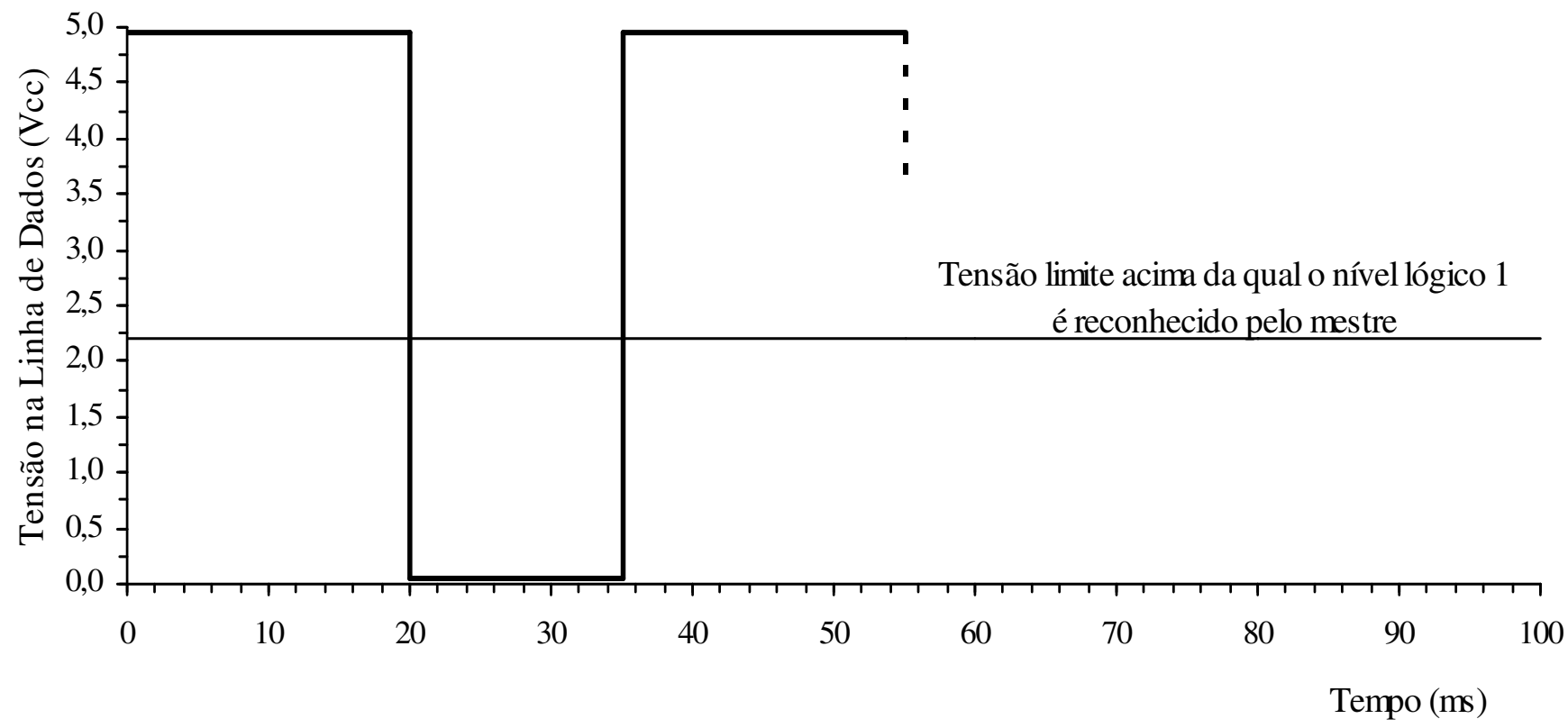

FIGURA 1. Transição entre níveis lógicos em condições ideais.

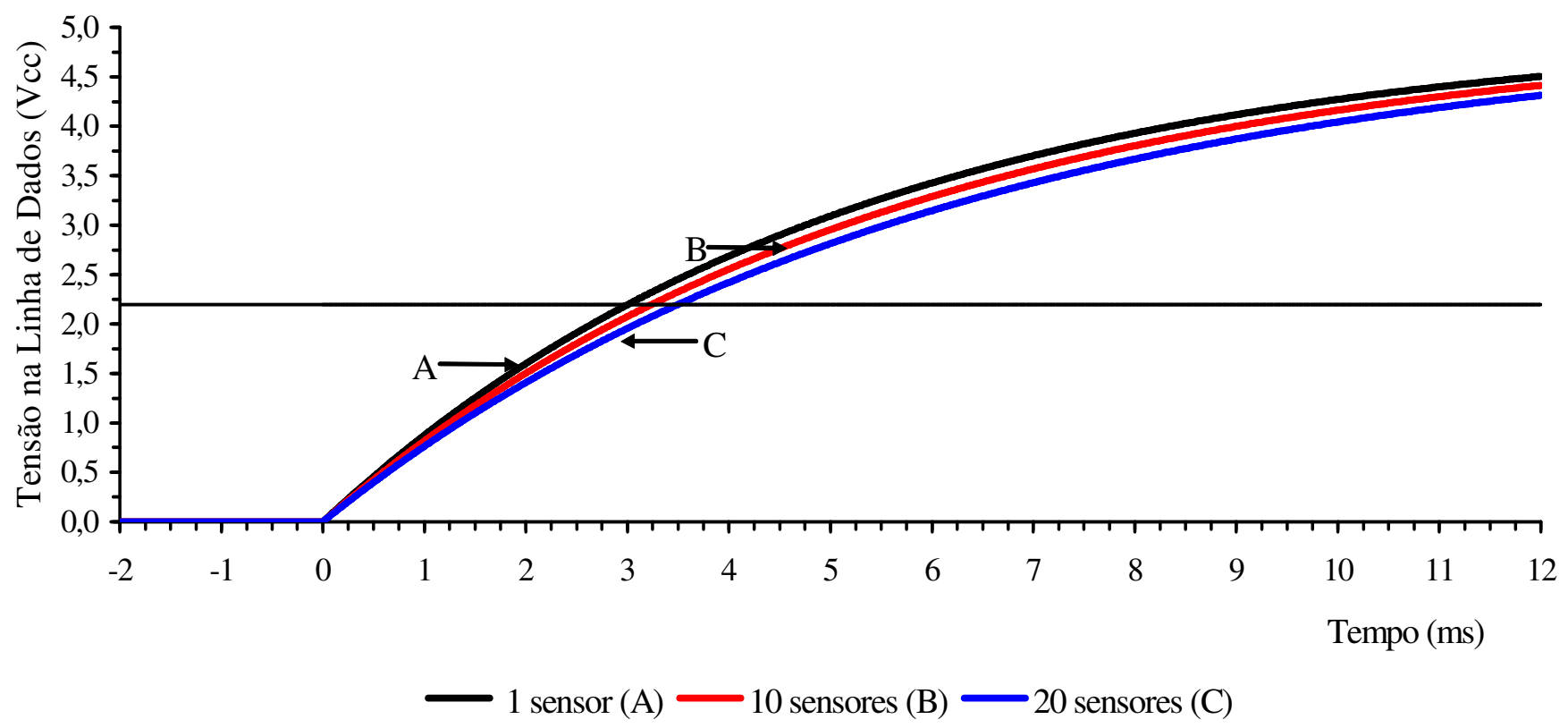

FIGURA 2. Transição do nível lógico 0 (zero) para o nível lógico 1 (um) obtido com as avaliações teóricas do cabo telefônico CCI com 1; 10 e 20 sensores DS1820. 


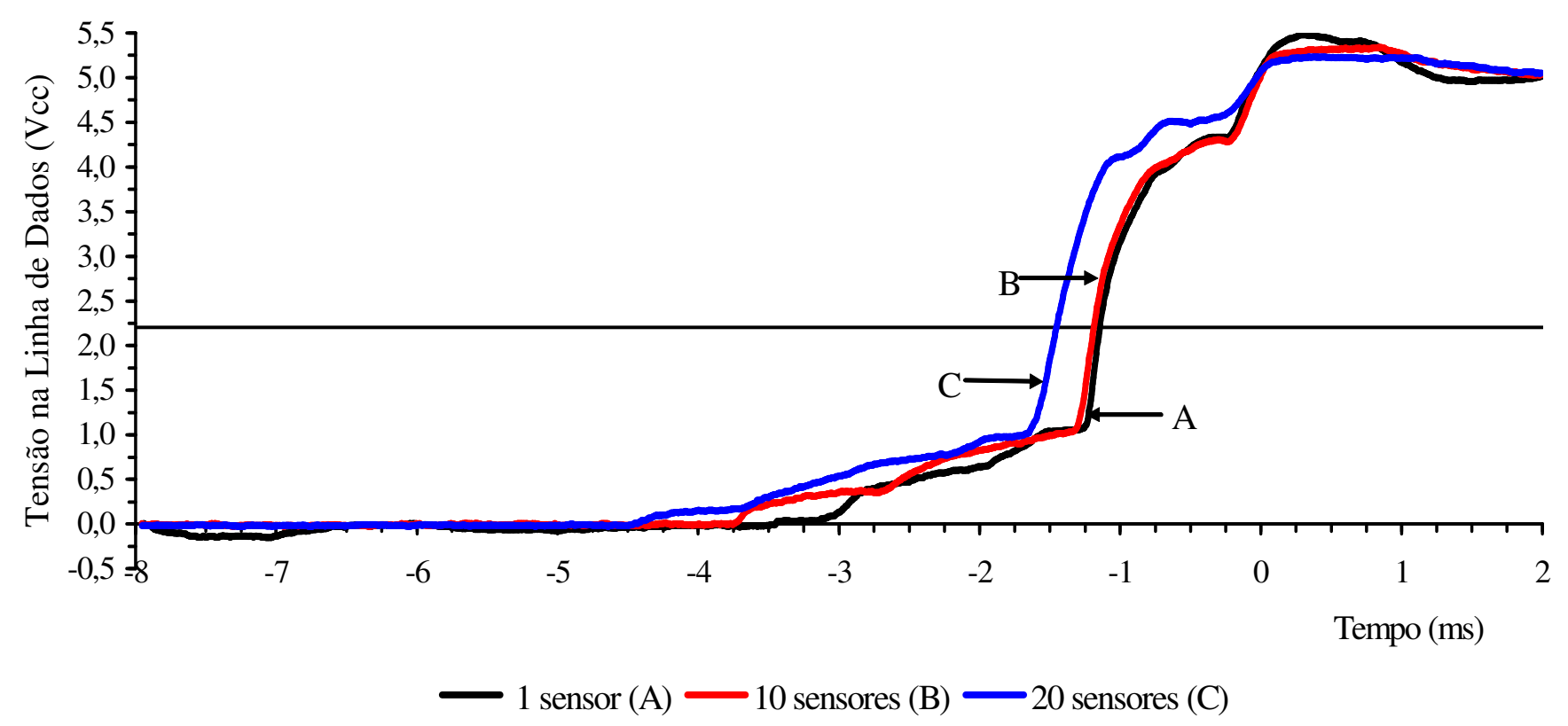

FIGURA 3. Transição do nível lógico 0 (zero) para o nível lógico 1 (um) obtido com as avaliações experimentais do cabo telefônico CCI com 1; 10 e 20 sensores DS1820.

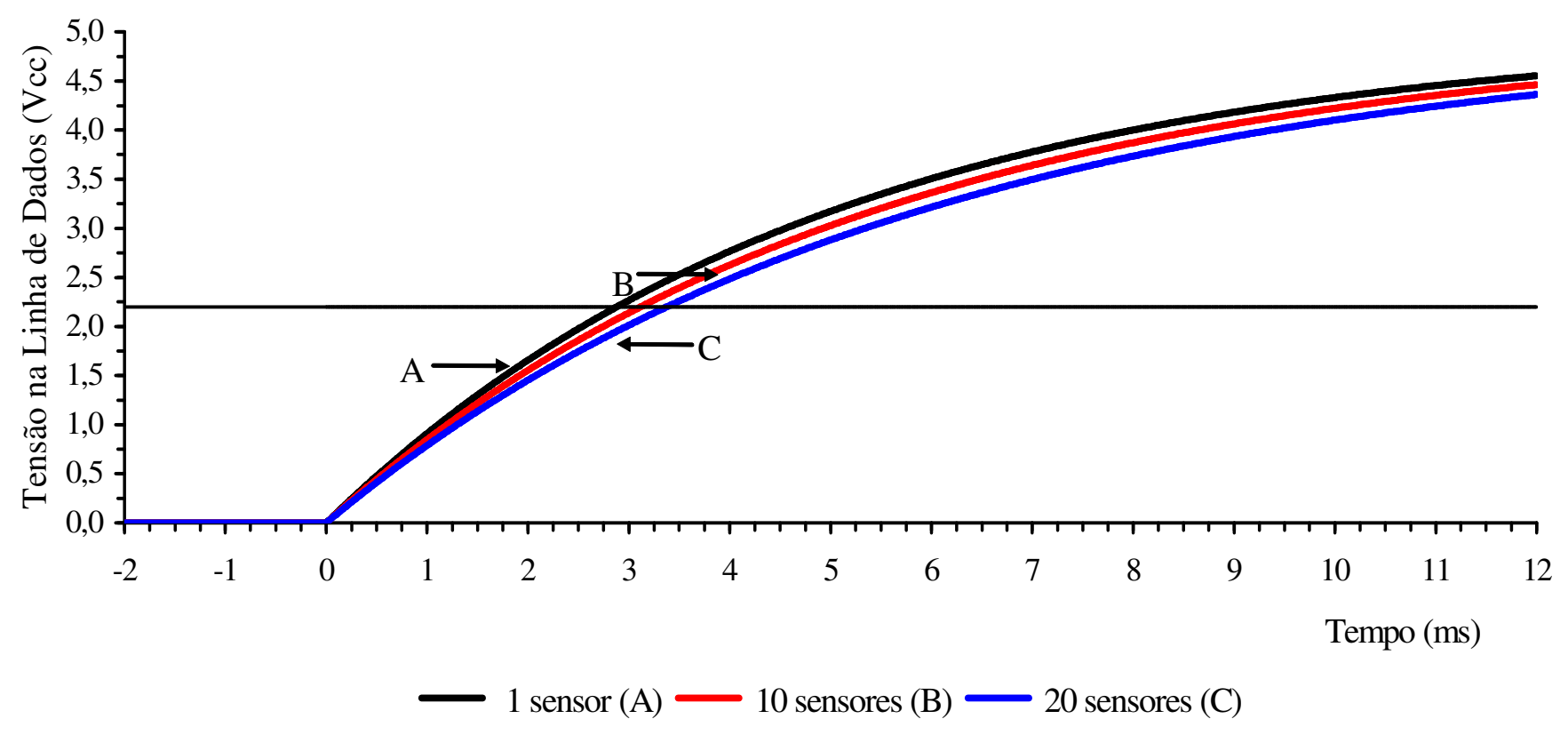

FIGURA 4. Transição do nível lógico 0 (zero) para o nível lógico 1 (um) obtido com as avaliações teóricas do cabo coaxial blindado com 1; 10 e 20 sensores DS1820. 


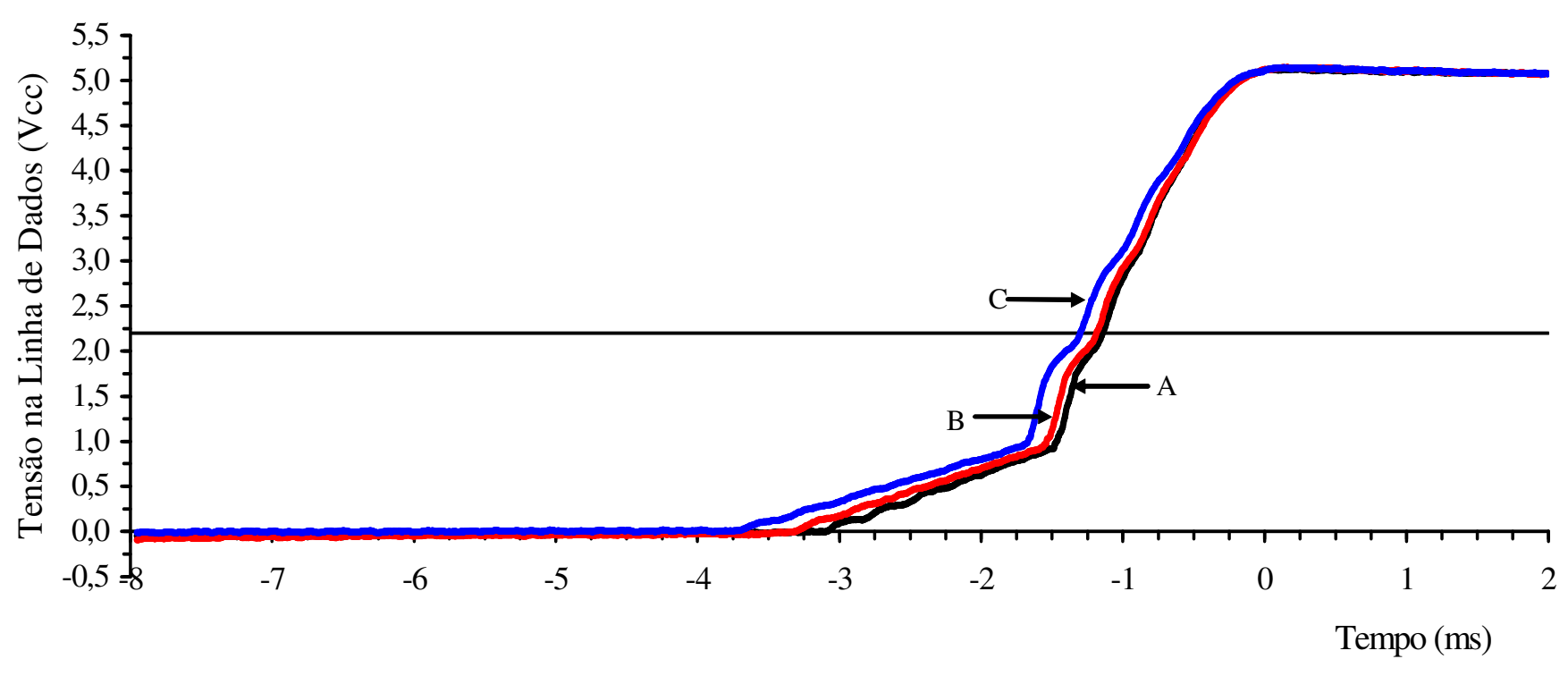

- 1 sensor $(\mathrm{A}) \longrightarrow 10$ sensores $(\mathrm{B})-20$ sensores $(\mathrm{C})$

FIGURA 5. Transição do nível lógico 0 (zero) para o nível lógico 1 (um) obtido com as avaliações experimentais do cabo coaxial blindado com 1; 10 e 20 sensores DS1820.

Com base nas Figuras 2 a 5 e nos resultados apresentados na Tabela 1, verifica-se, para cada um dos cinco tipos de condutores estudados, que na medida em que o número de sensores de temperatura DS1820 aumenta, o tempo de transição do nível lógico 0 (zero) para o nível lógico 1 (um) também aumenta. Esse comportamento pode ser explicado pelo acréscimo na capacitância total do sistema. Além disso, os tempos de transição teóricos obtidos a partir da eq.(1) sempre superestimam os resultados experimentais.

TABELA 1. Tempos de transição ( $\mu$ s) do nível lógico 0 (zero) para o nível lógico 1 (um) no sistema 1wire $^{\mathrm{TM}}$, obtidos com as avaliações teóricas e experimentais, para os cinco tipos de condutores com 1; 10 e 20 sensores de temperatura DS1820.

\begin{tabular}{lcccccc}
\hline & \multicolumn{3}{c}{ Avaliação Experimental } & \multicolumn{3}{c}{ Avaliação Teórica } \\
\hline \multicolumn{1}{c}{ Número de sensores } & 1 & 10 & 20 & 1 & 10 & 20 \\
\hline Cabo telefônico CCI* & 2,32 & 2,54 & 2,97 & 3,00 & 3,28 & 3,54 \\
Cabo telefônico trançado FI* & 2,54 & 2,63 & 2,90 & 3,22 & 3,43 & 3,69 \\
Cabo telefônico paralelo FI* & 2,62 & 2,77 & 2,98 & 4,03 & 4,32 & 4,53 \\
Cordão trançado** & 1,61 & 1,77 & 1,95 & 2,07 & 2,38 & 2,64 \\
Cabo coaxial blindado*** & 1,90 & 2,11 & 2,38 & 2,87 & 3,18 & 3,37 \\
\hline
\end{tabular}

* comprimento de $100 \mathrm{~m}$; ** comprimento de $60 \mathrm{~m}$; *** comprimento de $25 \mathrm{~m}$.

Na Tabela 2, apresentam-se os resultados gerais das avaliações experimentais da transmissão de dados de temperatura no sistema 1-wire ${ }^{\mathrm{TM}}$, no que se refere aos comprimentos limites, em função do tipo de condutor e do número de sensores de temperatura DS1820.

Com a finalidade de compensar o aumento na capacitância total do sistema 1-wire ${ }^{\mathrm{TM}}$, devido ao acréscimo do número de sensores DS1820, os comprimentos máximos dos condutores tiveram de ser reduzidos para que não houvessem interrupções na transmissão de dados. Essas reduções nos comprimentos máximos, conforme mostrado na Tabela 2, não foram idênticas, nem mesmo lineares, entre os cinco tipos de condutores estudados. Por exemplo, para o cabo telefônico CCI, o comprimento 
máximo foi reduzido de $150 \mathrm{~m}$ para $105 \mathrm{~m}$ quando o número de sensores de temperatura DS1820 aumentou de 1 para 20. Por outro lado, para o cabo coaxial blindado, essa redução no comprimento máximo não foi tão expressiva, passando de $34 \mathrm{~m}$ para $25 \mathrm{~m}$.

TABELA 2. Comprimentos máximos (m) para a transmissão de dados de temperatura no sistema 1wire $^{\mathrm{TM}}$ sem interrupção na comunicação entre o(s) sensor(es) DS1820 e o computador.

\begin{tabular}{lccc}
\hline \multicolumn{1}{c}{ Condutores } & 1 sensor & 10 sensores & 20 sensores \\
\hline Cabo telefônico CCI & 150 & 132 & 105 \\
Cabo telefônico trançado FI & 141 & 125 & 100 \\
Cabo telefônico paralelo FI & 140 & 125 & 100 \\
Cordão trançado & 100 & 80 & 60 \\
Cabo coaxial blindado & 34 & 30 & 25 \\
\hline
\end{tabular}

\section{CONCLUSÕES}

Os condutores telefônicos apresentaram os melhores desempenhos devido às suas características elétricas, principalmente a capacitância, serem mais favoráveis à transmissão de dados de temperatura por meio do sistema 1 -wire ${ }^{\mathrm{TM}}$.

Com relação aos efeitos do número de sensores de temperatura DS1820 na transmissão de dados, o aumento do número de sensores no sistema 1-wire ${ }^{\mathrm{TM}}$ causa a necessidade de reduções nos comprimentos máximos dos condutores, a fim de que não ocorram interrupções na comunicação.

O sistema 1-wire ${ }^{\mathrm{TM}}$ para aquisição de dados de temperatura mostrou-se ser um sistema de monitoramento automático adequado para aplicações agrícolas desde que os condutores sejam corretamente selecionados.

\section{REFERÊNCIAS}

AWTREY, D. Transmitting data and power over a one-wire bus. Sensors - The Journal of Applied Sensing Technology. Disponível em: http://www.advanstar.com. Acesso em: 10 nov. 2001.

CURTIS, S.E. Environmental management in animal agriculture. AMES: The Iowa State University, 1983. 409 p.

DALLAS SEMICONDUCTOR. DS1820: 1-wire digital thermometer. Disponível em: <http://www.maxim-ic.com> Acesso em: 22 nov. 2001.

DALLAS SEMICONDUCTOR. MicroLAN - In the long run. Application Note 108. Disponível em: <http://www.maxim-ic.com> Acesso em: 27 jan. 2002 a.

DALLAS SEMICONDUCTOR. Quick guide to 1-wire net using computers and microcontrollers. Application Note 132. Disponível em: <http://www.maxim-ic.com> Acesso em: 12 jun. 2002 b.

MONTEIRO, P.M.B. Tecnologia 1-wire ${ }^{T M}$ aplicada ao controle em tempo real de sistemas de aeração de grãos. 2002. 135 f. Tese (Doutorado em Energia na Agricultura) - Universidade Federal de Viçosa, Viçosa - MG, 2002.

STEIDLE NETO, A.J. Avaliação do sistema 1-wire ${ }^{T M}$ para aquisição de dados de temperatura em instalações agrícolas. 2003. 110 f. Dissertação (Mestrado em Construções Rurais e Ambiência) Universidade Federal de Viçosa, Viçosa - MG, 2003. 